Article

\title{
Assessing Reduction of Antibiotic Prescribing for Acute, Non- complicated Infections in Primary Care in Germany: Multi-step Outcome Evaluation in the Cluster-Randomized Trial ARena
}

Regina Poss-Doering ${ }^{1 *+}$, Dorothea Kronsteiner ${ }^{2+}$, Martina Kamradt ${ }^{1}$, Petra Kaufmann-Kolle ${ }^{3}$, Edith Andres ${ }^{3}$, Veit Wambach$^{4}$, Julian Bleek ${ }^{5}$, Michel Wensing ${ }^{1}$, ARena-study group ${ }^{* *}$, Joachim Szecsenyi ${ }^{1,3}$

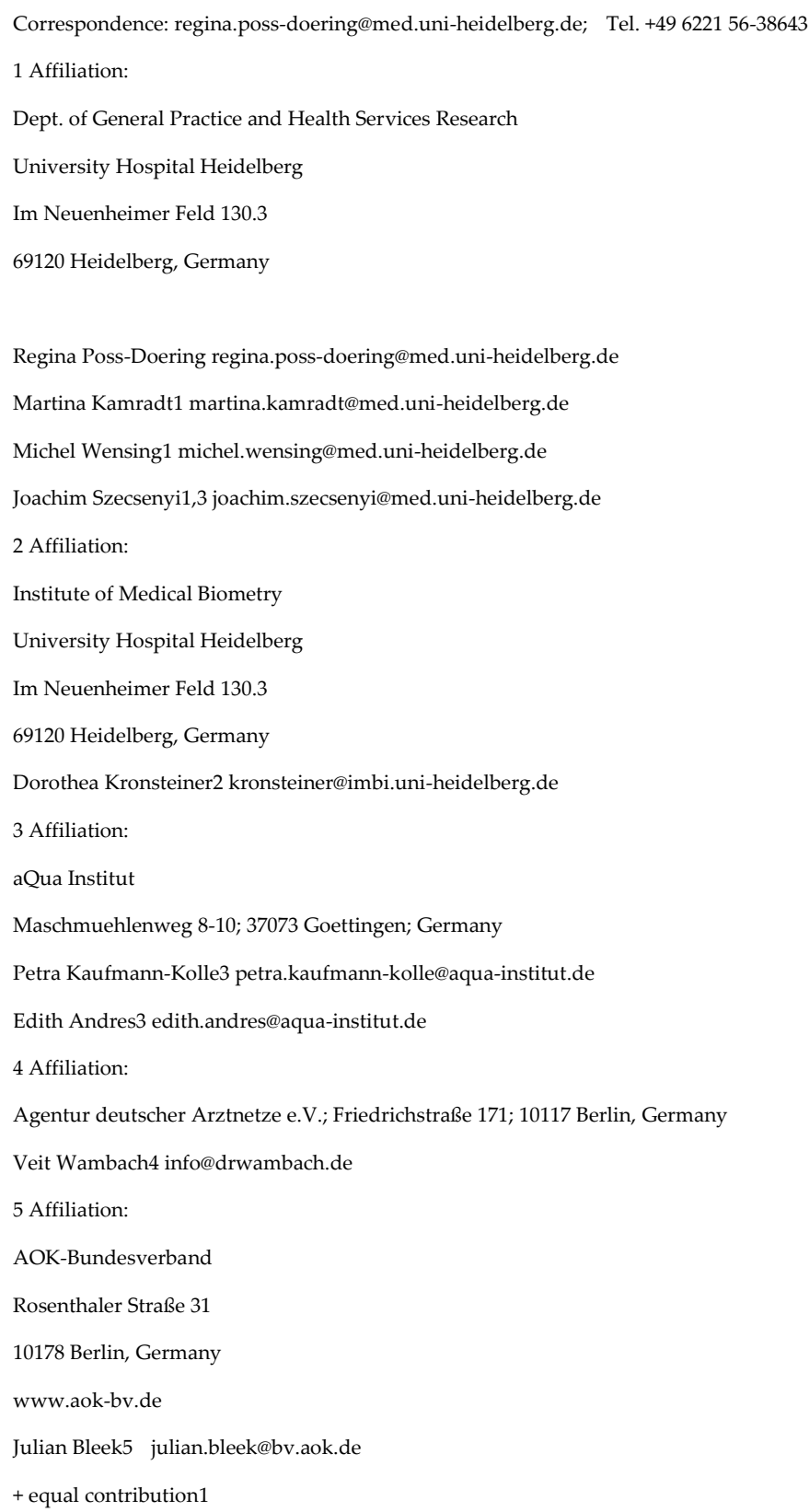

Abstract: The three-armed cluster-randomized trial ARena (Sustainable reduction of antibiotic-induced antimicrobial resistance) aimed to foster appropriate antibiotic use and reduce overprescribing in German ambulatory care to counter antibiotic resistance. Multi-faceted interventions targeted 
primary care physicians, teams and patients. This study examined effectiveness of the implementation program. ARena was conducted in 14 primary care networks with 196 practices. All arms received data-based feedback on antibiotics prescribing and quality circles. Arms II and III received different add-on components each. Primary outcome examined is the prescribing rate for systemic antibiotics for cases with non-complicated acute infections (upper respiratory tract, bronchitis, sinusitis, tonsillitis, otitis media). Secondary outcomes refer to prescribing of quinolones and guideline-recommended antibiotics. Based on pseudonymized quarterly claims data, mixed logistic regression models examined pre-post intervention antibiotic prescribing rate changes and compared to matched standard care. A significant rate reduction (arm I 11.7\%; arm II 9.9\%; arm III 12.7\%) and significantly lower prescribing rates were observed for all arms $(20.1 \%, 18.9 \%$ and $23.6 \%)$ compared to matched standard care $(29.4 \%)$. Fluoroquinolone prescribing was reduced in all intervention arms and rates for recommended substances generally increased. No significant post-interventional difference between intervention arms was detected. Findings indicate implementation program impact compared to standard care.

\section{Trial registration: ISRCTN, ISRCTN58150046}

Keywords: antimicrobial resistance; antibiotic prescribing; acute non-complicated infections; primary care; data-based feedback; mixed logistic regression model; multi-faceted intervention;

\section{Introduction}

On a global and on national scale, development and increase of antimicrobial resistance are advanced by over-consumption of antibiotics in human and veterinary healthcare and by inappropriate prescribing. In Germany, about $85 \%$ of the used antibiotics are prescribed during consultations in the primary care sector [1,2]. Compared to antibiotic consumption in other countries, the use of antibiotics in Germany is considered moderate $[3,4]$. However, there is a potential for a lowering of prescribing rates for specific conditions treated in primary care where antibiotics are prescribed by general practitioners in $41 \%$ of all consultations for acute respiratory tract infections (ARTI) [2] and only $52 \%$ of these prescriptions correspond to prevailing clinical recommendations [5].

In the context of the national strategy to reduce antibiotic consumption and counteract antimicrobial resistances [6], the ARena trial (Sustainable reduction of antibioticinduced antimicrobial resistance)was conducted as a (non-blinded) three-armed cluster randomized trial between 2017 and 2020. The aim of the trial was to examine the impact of a complex multifaceted implementation program intended to foster the rational use of antibiotics and reduce antibiotic prescribing for acute non-complicated infections of viral origin in primary care settings. The comprehensive program was developed in collaboration with the participating primary care networks (PCNs) and based on relevant published research identified in a literature review [7-11]. PCNs can be understood as formalized collaborations of healthcare providers who interact on a regular basis, standardize care, share patients, discuss concerns, support practice management and attend continuing education [12]. Such collaborative healthcare provider networks support coordination of care and contribute to improvements in care quality and safety [13]. Thus, they were expected to amplify the impact of the implementation program.

The ARena implementation program used multi-faceted interacting components to address knowledge, attitudes and experiences of primary care physicians (General Practitioners, Gynecologists, Otolaryngologists, Urologists, and Pediatricians), care teams and patients about the use of antibiotics in PCNs across two German federal states (Bavaria and North Rhine-Westphalia). The program used different strategies to reduce 
undesirable prescribing rates. All three intervention arms received a base module of intervention components comprising e-learning on communication to limit misinterpretation of patient preferences, moderated quality circles with data-based feedback on antibiotics prescribing for physicians to facilitate appropriate prescribing, public information campaigns including social media, performance-based additional reimbursement and culture-sensitive print information material for patients. Based on program developers' assessment of barriers for implementation, arm II received e-learning on communication and separate QCs addressing practice teams, and tablet pcs providing patient information in waiting areas. Sensitizing practice teams for a stronger interaction with patients might facilitate a relieve of the physician's workload and contribute to optimized decision-making [14, 15], thus including the team was assumed to be supportive for rational prescribing. Considering the developments in German healthcare, add-ons in arm III were future-orientated and comprised of multidisciplinary, cross-sectoral quality circles and a computerized decision support system (CDSS) to be used in the practice admin system. Current evidence of all base module intervention components validated positive effects on physician prescribing [9, 16-18]. Evidence regarding effects of add-on components in arms II and III was still limited [16, 19, 20], or inconclusive [16, 21, 22]. Nevertheless, effects were expected to be higher in arms II and III than in arm I since add-ons targeted specific barriers for change and were assumed to be promising tools for reduction of errors in medical decision-making [23]. A more detailed description of the study design and intervention components used can be found elsewhere [24].

Primary objective of this study was to examine and compare the change of prescribing rates for targeted index diagnoses in the ARena intervention arms and compare prescribing rates to non-participating standard care, matched to participating PCNs based on quarterly claims data.

\section{Results}

A total of 87.377 patient cases with ARena index diagnoses during the intervention period (Q4 2017-Q2 2019) were included for analysis which exceeded the target of 79.685 cases calculated for the evaluation. Sample sizes differ between primary and secondary outcomes, because they focus on subgroups of the primary outcome population. One patient might represent several cases.

Physicians working in general practice were the largest group of prescribers in PCNs $(76.5 \%)$ and in standard care $(75.8 \%)$ (Appendix A, Supplementary File 1, Table A1 details medical specialty groups distribution). Study populations differed per indexed consultation reason regarding number of cases and age of patients. The sample sizes in the three intervention arms were as follows: in intervention arm I pre 9,673 and post 10,143 cases, in intervention arm II pre 4,583 and 6,730 post cases, and in intervention arm III pre 3,951 and post 5,076 cases. For the matched standard care cohort, 25,385 cases pre and 25,144 post intervention were included. Patient characteristics for the included cases are presented in Table 1 (see Appendix A, Supplementary File 1, Table A2 for a detailed description pre- and post-intervention). 


\section{Intervention}

(cases)
Matched standard

care

(no intervention)

\begin{tabular}{|c|c|c|c|c|c|}
\hline & & $\begin{array}{l}\text { Arm I } \\
\qquad(n=10,143)\end{array}$ & $\begin{array}{l}\text { Arm II } \\
\qquad(n=6,730)\end{array}$ & $\begin{array}{l}\text { Arm III } \\
\qquad(n=5,076)\end{array}$ & $(n=25,144)$ \\
\hline \multirow[t]{3}{*}{ Age, $n(\%)$} & $<18$ & $578 \quad(5.7)$ & $757 \quad(11.2)$ & $143(2.8)$ & $4,210(16.7)$ \\
\hline & $18-65$ & 7,869 (77.6) & $4,684(69.6)$ & $3,910(77.0)$ & $18,960(75.4)$ \\
\hline & $>65$ & 1,696 (16.7) & $1,289(19.2)$ & $1,023(20.2)$ & $1,974(7.9)$ \\
\hline Gender, n (\%) & Male & $4,405(43.4)$ & $2,900(43.1)$ & $2,074(40.9)$ & $11,891(47.3)$ \\
\hline Nationality, n (\%) & German & 9,115 (89.9) & $5,425(80.6)$ & $4,144(81.6)$ & $18,681(74.3)$ \\
\hline \multirow[t]{4}{*}{$\mathrm{CCl}, \mathrm{n}(\%)$} & 0 & $5,734(56.5)$ & $3,858(57.3)$ & $2,604(51.3)$ & $17,412(69.2)$ \\
\hline & 1,2 & $2,926(28.8)$ & 1,945 (28.9) & 1,609 (31.7) & $6,259(24.9)$ \\
\hline & 3,4 & $781(7.7)$ & $453(6.7)$ & $492(9.7)$ & $830(3.3)$ \\
\hline & $\geq 5$ & $702(6.9)$ & $474(7.0)$ & $371(7.3)$ & $643(2.6)$ \\
\hline \multirow[t]{5}{*}{ Index diseases, n (\%) } & Bronchitis & $2,442(24.1)$ & $1,569(23.3)$ & $1,457(28.7)$ & $5,796(23.1)$ \\
\hline & URTI & $7,620(75.1)$ & $4,916(73.0)$ & 3,719 (73.3) & $17,663(70.2)$ \\
\hline & Sinusitis & $962(9.5)$ & $867(12.9)$ & $700(13.8)$ & $1,642(6.5)$ \\
\hline & Tonsillitis & $507(5.0)$ & $335(5.0)$ & $220(4.3)$ & $1,654(6.6)$ \\
\hline & Otitis Media & $474(4.7)$ & $357(5.3)$ & $263(5.2)$ & $1,809(7.2)$ \\
\hline
\end{tabular}

CCI: Charlson Comorbidity Index; URTI: Upper respiratory tract infection

\subsection{Primary outcome}

Intervention arms showed different baseline prescribing rates and post-intervention prescribing rate reductions. A significant reduction of antibiotic prescriptions was observed in intervention arm II (post versus pre: $\mathrm{OR}=0.547$ 95\%-CI=[0.493; 0.607], $\mathrm{p}<0.001$ ) and in intervention arm III (post versus pre: $\mathrm{OR}=0.519$ 95\%-CI=[0.467; 0.576], $\mathrm{p}<0.001$ ). Also, in intervention arm I, the pre-post-comparison showed a significant reduction of antibiotic prescriptions $(\mathrm{OR}=0.52395 \%-\mathrm{CI}=[0.485 ; 0.563], \mathrm{p}<0.001)$. No significant difference was detected between intervention arms regarding within-group change in the RCT (II vs. I: $0.86395 \%$-CI=[0.658; 1.13] p=0.284; III vs. I: 1.182 95\%-CI=[0.895;1.561], p=0.239; II vs. III: $1.01995 \%-\mathrm{CI}=[0.781 ; 1.331], \mathrm{p}=0.888)$. Figure 1 illustrates the primary outcome as percentages of cases with index diseases in the intervention arms and matched standard care (pre vs. post). 


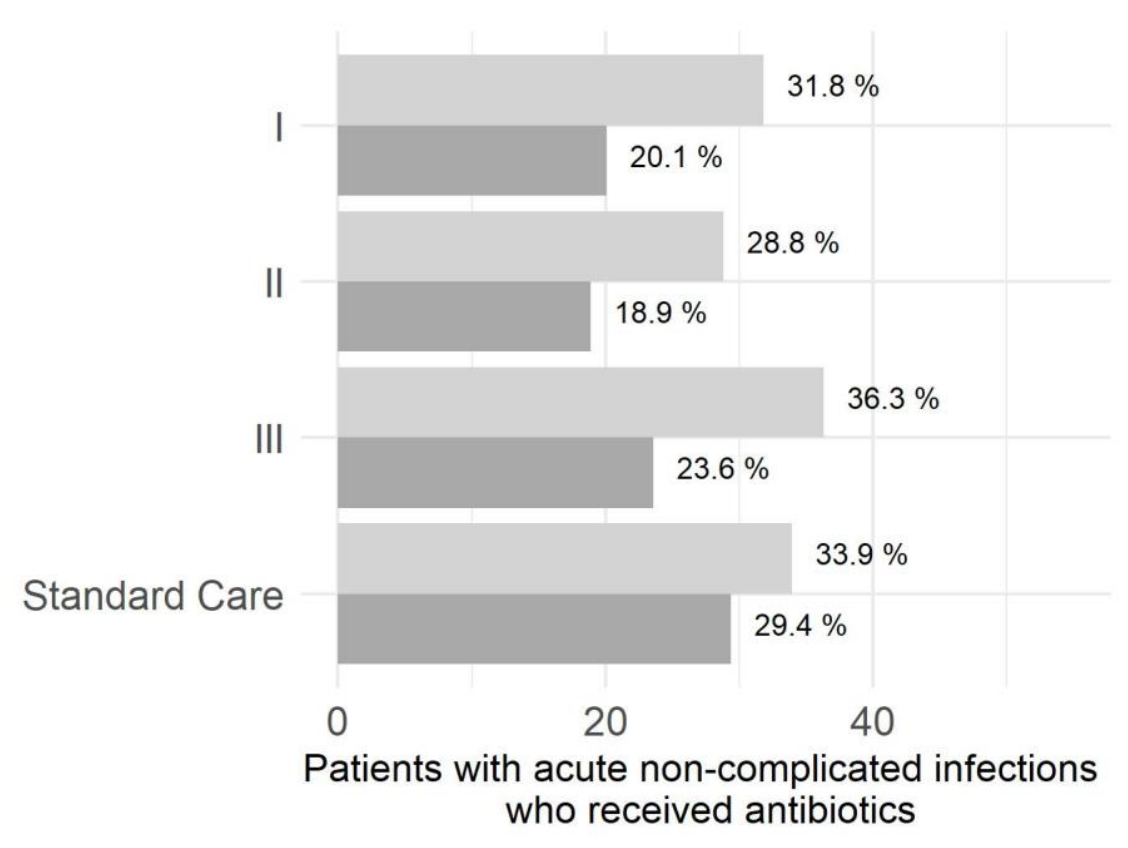

pre

post

$\mathrm{I}=$ intervention arm 1; II = intervention arm II; III = intervention arm III

Figure 1: Percentage of patient cases who consulted primary care practices with acute noncomplicated infections (index diseases) and were treated with systemic antibiotics (primary outcome)

The comparison of changes in each of the three intervention arms to the matched standard care was evaluated in logistic mixed regression models and considered pre/post intervention as covariate. For all intervention arms, a larger reduction of antibiotic prescribing compared to the matched standard care was observed $(11,7 \%$ in arm I vs. $4,5 \%$ in standard care: $\mathrm{OR}=0,59695 \%-\mathrm{CI}=[0,572 ; 0,621], \mathrm{p}<0.001 ; 9,9 \%$ in arm II vs. $4,5 \%$ in standard care: $\mathrm{OR}=0,66195 \%-\mathrm{CI}=[0,629 ; 0,695], \mathrm{p}<0.001 ; 12,7 \%$ in III vs. $4,5 \%$ in standard care: $\mathrm{OR}=0,72695 \%-\mathrm{CI}=[0,689 ; 0,764], \mathrm{p}<0.001)$ ). Table 2 describes the pre- post comparison of antibiotic prescribing rates, as well as the pre-post comparison between intervention arms and Table 3 the comparison to the matched standard care adjusted for additional covariates.

Table 2: Comparison of antibiotic prescribing rates (pre vs. post, primary outcome) and comparison between intervention arms 


\begin{tabular}{|c|c|c|c|c|c|}
\hline \multirow{5}{*}{ Intervention arm I } & & & lower & upper & \\
\hline & post vs pre & 0.523 & 0.485 & 0.563 & $<0.001$ \\
\hline & Female vs male & 1.274 & 1.179 & 1.376 & $<0.001$ \\
\hline & Age $<18$ vs $18-65$ & 0.675 & 0.559 & 0.815 & $<0.001$ \\
\hline & Age $>65$ vs $18-65$ & 1.324 & 1.193 & 1.469 & $<0.001$ \\
\hline \multirow[t]{4}{*}{ Intervention arm II } & post vs pre & 0.547 & 0.493 & 0.607 & $<0.001$ \\
\hline & Female vs male & 1.371 & 1.227 & 1.532 & $<0.001$ \\
\hline & Age $<18$ vs $18-65$ & 0.64 & 0.412 & 0.994 & 0.047 \\
\hline & Age >65 vs $18-65$ & 1.167 & 1.008 & 1.351 & 0.038 \\
\hline \multirow[t]{4}{*}{ Intervention arm III } & post vs pre & 0.519 & 0.467 & 0.576 & $<0.001$ \\
\hline & Female vs male & 1.296 & 1.164 & 1.443 & $<0.001$ \\
\hline & Age $<18$ vs $18-65$ & 0.676 & 0.494 & 0.925 & 0.014 \\
\hline & Age >65 vs $18-65$ & 1.479 & 1.299 & 1.684 & $<0.001$ \\
\hline \multirow{7}{*}{$\begin{array}{l}\text { Comparison between inter- } \\
\text { vention arms }\end{array}$} & Intervention arm II vs I & 0.863 & 0.658 & 1.13 & 0.284 \\
\hline & Intervention arm III vs I & 1.019 & 0.781 & 1.331 & 0.888 \\
\hline & Intervention arm III vs II & 1.182 & 0.895 & 1.561 & 0.239 \\
\hline & post vs pre & 0.561 & 0.535 & 0.589 & $<0.001$ \\
\hline & Female vs male & 1.274 & 1.214 & 1.338 & $<0.001$ \\
\hline & Age $<18$ vs $18-65$ & 0.683 & 0.595 & 0.784 & $<0.001$ \\
\hline & Age $>65$ vs $18-65$ & 1.255 & 1.179 & 1.337 & $<0.001$ \\
\hline
\end{tabular}

Adjusting the primary outcome model for further patient and practice characteristics indicated that the difference between interventions arms and matched standard care remained significant. Female gender, age, and Charlson index were associated with a higher likelihood for an antibiotic prescription. Specialists prescribed less antibiotics compared to general practitioners. Table 3 details the comparison of intervention arms to the matched standard care adjusted for additional covariates.

Table 3: Comparison of antibiotic prescribing rates in intervention arms to matched standard care (pre vs. post, primary outcome) adjusted for additional covariates 


\begin{tabular}{|c|c|c|c|c|c|}
\hline \multirow{15}{*}{$\begin{array}{l}\text { Comparison to standard } \\
\text { care }\end{array}$} & Intervention arm I vs standard care & 0.596 & 0.572 & 0.621 & $<0.001$ \\
\hline & $\begin{array}{l}\text { Intervention arm II vs standard } \\
\text { care }\end{array}$ & 0.661 & 0.629 & 0.695 & $<0.001$ \\
\hline & $\begin{array}{l}\text { Intervention arm III vs standard } \\
\text { care }\end{array}$ & 0.726 & 0.689 & 0.764 & $<0.001$ \\
\hline & post vs pre & 0.699 & 0.679 & 0.721 & $<0.001$ \\
\hline & Female vs male & 1.198 & 1.162 & 1.234 & $<0.001$ \\
\hline & Age $<18$ vs $18-65$ & 0.54 & 0.503 & 0.579 & $<0.001$ \\
\hline & Age $>65$ vs $18-65$ & 0.836 & 0.778 & 0.899 & $<0.001$ \\
\hline & $\mathrm{CCl} 1,2$ vs 0 & 1.584 & 1.531 & 1.638 & $<0.001$ \\
\hline & $\mathrm{CCl} 3,4$ vs 0 & 1.603 & 1.498 & 1.716 & $<0.001$ \\
\hline & $\mathrm{CCl}>=5$ vs 0 & 1.503 & 1.395 & 1.62 & $<0.001$ \\
\hline & Northern Europe vs German & 0.916 & 0.74 & 1.128 & 0.417 \\
\hline & $\begin{array}{l}\text { Eastern Europe, Turkey, Arabic } \\
\text { states vs German }\end{array}$ & 0.978 & 0.935 & 1.022 & 0.321 \\
\hline & Other vs German & 0.818 & 0.719 & 0.929 & 0.002 \\
\hline & Southern Europe vs German & 1.087 & 0.994 & 1.189 & 0.066 \\
\hline & urban vs rural & 0.736 & 0.712 & 0.761 & $<0.001$ \\
\hline
\end{tabular}

CCI: Charlson Comorbidity Index; vs: versus

Northern Europe, Eastern, Europe, Southern Europe, Other: Nationality

\subsection{Secondary outcomes}

For the secondary outcome (SO) of fluoroquinolone prescribing, low pre-interventional rates were observed for trial participants and these were further reduced during the intervention period (for details see Appendix A, Supplementary File 2, Table A3). At the same time, the prescribing rate of fluoroquinolones decreased in standard care as well. The comparison between intervention arms and matched standard care showed that fluoroquinolone prescribing was lower in all intervention arms (I vs. standard care: $\mathrm{OR}=0.911$ $95 \%-\mathrm{CI}=[0.811 ; 1.021]$; II vs. standard care: $\mathrm{OR}=0.7195 \% \mathrm{CI}=[0.594 ; 0.844]$; III vs. standard care: $\mathrm{OR}=0.716[0.613 ; 0.833])$.

Prescribing rates for guideline-recommended substances were evaluated for each index disease separately. The highest prescribing rate of recommended substances for URTI was observed at baseline (pre-intervention) in matched standard care. For the intervention arms, a large increase was observed post intervention when rates are higher compared to matched standard care (I vs. standard care: OR=1.615 95\%-CI=[1.435;1.816]; II vs. standard care: $\mathrm{OR}=1.456 \quad 95 \%-\mathrm{CI}=[1.254 ; 1.687] ; \quad$ III $\quad$ vs. standard care: $\mathrm{OR}=1.539 \quad 95 \%$ $\mathrm{CI}=[1.336 ; 1.769])$.

For bronchitis, an increase in prescribing of recommended substances was recognized in all intervention arms and in matched standard care. Intervention arms I and III 
reached a substantially higher rate compared to standard care (I vs. standard care: $\mathrm{OR}=1.634$ 95\%-CI=[1.439;1.855]; II vs. standard care: OR=0,987 95\%-CI=[0,83;1,17]; III vs. standard care: $\mathrm{OR}=1.37495 \%-\mathrm{CI}=[1.181 ; 1.594])$. For sinusitis, all intervention arms showed higher post-interventional prescribing of recommended antibiotics (I vs. standard care: $\mathrm{OR}=1.33895 \%-\mathrm{CI}=[1.057 ; 1.691]$; II vs. standard care: $\mathrm{OR}=1.372$ 95\%-CI=[1.012;1.851]; III vs. standard care: $\mathrm{OR}=2.198$ 95\%-CI=[1.685;2.859]). Patients suffering from tonsillitis in intervention arm I had the highest chance of receiving a recommended substance for the pre timepoint (33.5\% recommended antibiotics) while the highest absolute increase was observed in intervention arm III (pre $10.7 \%$, post $25.7 \%$ ). Comparing post interventional prescribing, only intervention arm I shows a higher prescribing rate of recommended antibiotics compared to matched standard care (I vs. standard care: OR=1.157 95\%$\mathrm{CI}=[0.95 ; 1.406]$; II vs. standard care: $\mathrm{OR}=0.682$ 95\%-CI=[0.535;0.864]; III vs. standard care: $\mathrm{OR}=0.685$ [0.503; 0.919]). The highest prescribing rates of antibiotics recommended for otitis media are $41.5 \%$ of observed cases in intervention arm II post the intervention period (pre: $33.3 \%$ ) and $37.9 \%$ in intervention arm I (pre: $27.7 \%$ ), compared to $29.3 \%$ in matched standard care (pre: $23.6 \%$ ).

Figure 2 illustrates the percentage of patient cases who consulted participating PCN practices for one of the index diseases and received fluoroquinolones or were treated with guideline-recommended systemic antibiotics. For fluoroquinolone prescribing, a decrease, for recommended antibiotics, an increase in prescribing was targeted. Appendix A, Supplementary File 2, Table A3 describes the observed changes in recommended antibiotics prescribing regarding the examined index diagnoses and indicates the size of the respective patient populations. Comparison of the intervention arms and to matched standard care regarding the SOs is detailed in Appendix A, Supplementary File 2 Table A4. 
so 1

Standard Care

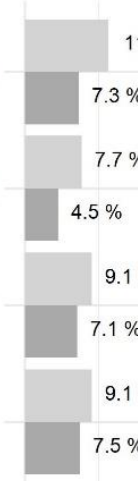

0

20

40

Patients with Index diagnosis and antibiosis who received fluoroquinolones

SO 3

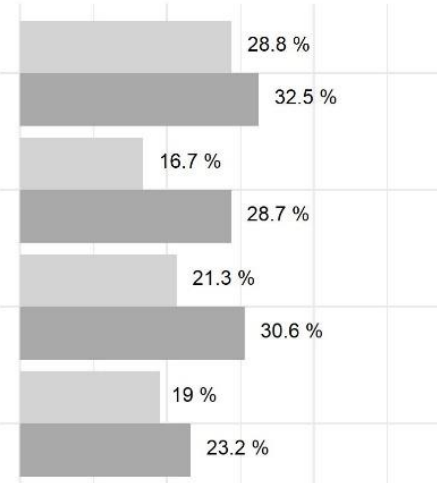

0

20

40

Patients with Bronchitis and antibiosis who received recommended antibiotics

\section{SO 5}

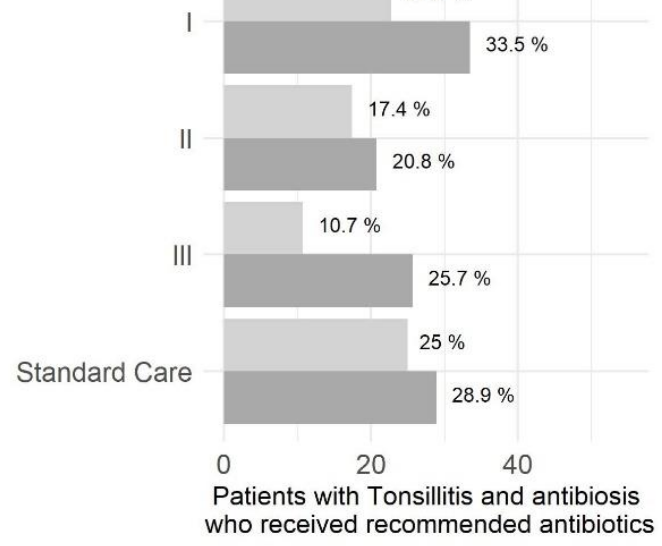

$\mathrm{SO}=$ Secondary Outcome

\section{SO 2}

Standard Care

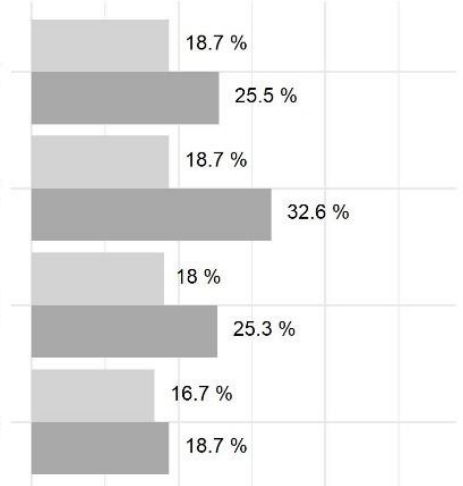

0

20

40

Patients with URTI and antibiosis who received recommended antibiotics

\section{SO 4}

Standard Care

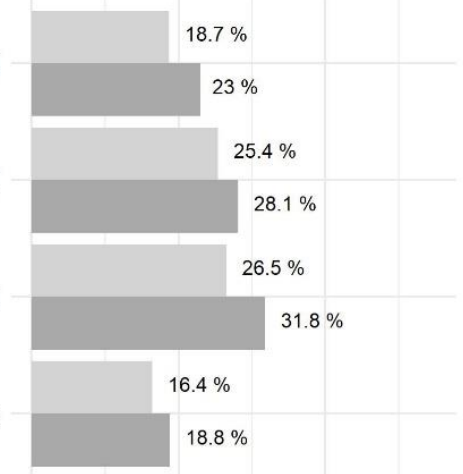

0

20

40

Patients with Sinusitis and antibiosis who received recommended antibiotics

\section{SO 6}

Standard Care

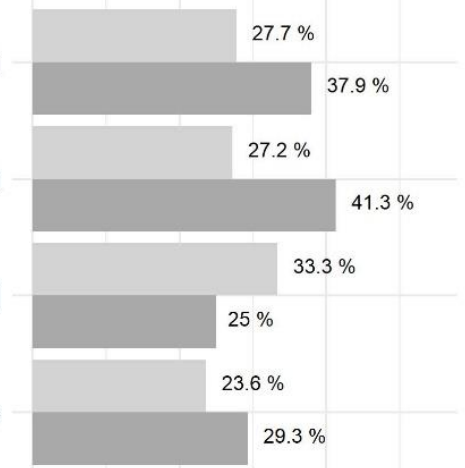

0

20

40

Patients with Otitis Media and antibiosis who received recommended antibiotics

pre

post

Figure 2: Proportion of patients who consulted primary care practices with acute non-complicated infections (index diseases) and received fluoroquinolones or were treated with recommended systemic or local antibiotics. 


$$
\mathrm{a}=1,
$$

\section{Discussion}

The implementation program focused on reduction of antibiotic prescribing in primary care in Germany for non-complicated self-limiting infections without indication for antibiotics. A significant reduction was observed in the pre-post comparison of all intervention arms. No significant difference between the randomized intervention arms was found, so add-on intervention components in arms II and III did not increase the effect. However, the implementation program was associated with lower antibiotic prescribing than in matched standard care, suggesting that it had impact on professional practice.

Different strategies were used to aim for a reduction of undesirable prescribing including enabling measures such as feedback reports, thematically focused quality circles and e-learning modules on physician-patient communication. Enabling measures have been shown to be associated with better acceptance and combining them with restricting interventions was associated with sustainability of the latter [25]. For the ARena trial, a combination of enabling and potentially restrictive intervention components was applied. Effects were expected to be higher in intervention arms II and III than in arm I since the add-on components targeted specific barriers for change (arm II) and match with overall trends in healthcare (arm III) . Enabling intervention components in arm II were not only targeted at prescribing physicians directly, but also addressed the practice teams, assuming their role in ambulatory care could be supportive for reduced prescribing by transferring knowledge and awareness to patients. Findings of the process evalaution conducted alongside ARena indicate that physicians in arm II viewed the additional components and particularly the involvement of the practice teams as very valuable since they perceived an increased health literacy among the more sensitized patients and a decrease in patient demand for antibiotics [26-28]. Providing health-related knowledge to patients via a tablet pc app however, was perceived to potentially discriminate against older patients with a lesser digital affinity, pose hygiene-related challenges in the practice and increase the practice team's workload. These factors, and a general concern about potential theft of the provided tablet pcs, accounted for a somewhat reduced intervention fidelity regarding this component in arm II. [26]. Combined with an acknowledged lower than initially expected patient demand for antibiotics, this might explain why the estimated potential impact on prescribing could not be observed.

In intervention arm III, the add-on components related to interprofessional care and the increased relevance of information technology solutions in healthcare. PCNs participating in arm III offered cross-sectoral QCs on own initiative and due to internal factors, not all initially planned meetings could take place. The CDSS offered in arm III was implemented into administrative practice software to provide adequate prompts when index diagnoses were coded, which could be ignored or turned off. Its implementation was delayed due to technical difficulties resulting in a shortened usage period for less practices than expected [26]. The process evaluation found that practices participating in arm III received new impulses from the interdisciplinary QCs and considered the CDSS a support for the integration of knowledge into daily care routines and the choosing of indicationappropriate antibiotics. However, a lower intervention fidelity regarding these two addon components caused by delayed or incomplete implementation may be typical for newer approaches and might illustrate why the expected additional effect could not be detected.

The trial was conducted in the particular setting of PCNs. Prior research had concluded that such collaborative health professional networks can contribute to improving healthcare quality and safety [29] and interventions delivered by peer community can be 
expected to be more successful than those delivered by agencies less connected to program recipients [30]. Thus, the PCNs were expected to be an enabling factor for change of prescribing routines. This was also supported by process evaluation findings [26, 31] which confirmed that it was an appropriate choice to conduct the ARena trial in the setting of PCNs. Observed antibiotic prescribing in the intervention arms at baseline [32] was both, lower than expected and lower than in matched standard care. It also differed between the three arms, yet could be reduced significantly. As prescribing rate reduction effects tend to be stronger when initial rates are high, this can be seen as confirmation for appropriateness of the study setting and the chosen improvement program.

For the $\mathrm{SO}$ of fluoroquinolone prescribing, the moderate prescribing still declined in all interventions arms as well as in the matched standard care. However, the chance of inappropriate antibiotic prescribing was substantially lower for cases in all intervention arms compared to cases in the matched standard care. Prescribing of guideline-oriented recommended antibiotics for the observed index diseases (URTI, bronchitis, sinusitis, tonsillitis, otitis media) improved in the intervention arms and were higher than observed prescribing in matched standard care. Exception here was prescribing in intervention arm III for otitis media which is a very common disease in early childhood [33,34] with antibiotics prescribed in many cases [35], although evidence does not support this for most children with mild disease [36-38]. It can be assumed that antibiotic prescriptions for otitis media might be written mainly by pediatricians who were underrepresented in the ARena intervention arms and hence the matched standard care. This potentially explains prescribing of recommended antibiotics remaining somewhat low since beside a nationwide decline in antibiotic prescribing to primary care patients in all age groups between 2010 and 2018, strong improvements and sustainable change in pediatric prescribing patterns have been identified [39].

Current guidelines in Germany recommend to consider antibiotic treatment for noncomplicated infections in patients with co-morbidities. Prescribing rates of recommended antibiotics increased in all intervention arms, but compared to matched standard care, the likelihood for patients with acute tonsillitis to receive a guideline-recommended antibiotic was lower in intervention arms II and III. Prior research indicated that a physician's personal attitude might be a determining factor in whether a patient receives an antibiotic prescription, or not [40]. A recent study evaluated characteristics of high and low prescribers of antibiotics in German primary care by examining routinely collected claims data . The study confirmed previous findings of considerable differences in prescribing rates between physicians and concluded that due to the limitations of secondary data, further research including the linkage of primary and secondary data should aim to determine causal relationships [41]. However, the discrepancy in our findings cannot be explained by the analysis, yet among possible reasons could also be diagnostic uncertainties referring to younger patients or recurrent incidence of the disease, known allergies in patients, or regional variations.

\section{Strengths and limitations}

Particular strengths of this study are the randomized evaluation design, high validity of claims data, low drop out of participating physicians, thorough exclusion of cases with diagnoses that warranted antibiotic therapy and the additional detailed analysis referring to the type of antibiotics (recommended, quinolones). All cases meeting the inclusion/exclusion criteria were considered. The main limitation of the randomized trial was the randomization at a high aggregation level (14 PCNs) with a risk of by-chance differences. In the observational comparison, the intervention arms were compared to matched standard care, not to a control group of practices from the participating PCNs since the network structure and peer exchange likely would have led to contamination. At pre-intervention, observed prescribing rates differed between the arms and were lower than expected. Limitations also include the absence of blinding and health-related outcome measures, and the restriction on ICD-10 codes and claims-related health insurance data 
where disease, patient, and practice information were limited. Direct connection between ICD-10 code and antibiotic prescription was not possible via the provided claims data. Therefore, ICD-10 codes and antibiotic prescription were matched by quarter which introduces a potential bias.

\section{Materials and Methods}

Study design

ARena was conducted as a prospective (non-blinded) three-armed cluster randomized trial in two German federal states (Bavaria and North Rhine-Westphalia) with fourteen PCNs as units of randomization. The study was conducted with an intervention period of 21 months, and two parts of evaluation: (a) an outcome evaluation based on quarterly claims-data provided by the statutory health insurance provider AOK and (b) a process evaluation based on surveys [24]. Examined were the comparison of baseline and post-interventional prescribing rates, and differences between the intervention arms and the matched cohort of non-participant matched standard care generated from claims data.

Study population

Fourteen PCNs in Bavaria (12 PCNs) and North Rhine-Westphalia (2 PCNs) were recruited by the aQua Institut, Göttingen, Germany, for participation in the ARena trial and randomized into arm I (4 PCNs), arm II (5 PCNs), and arm III (5 PCNs) by a statistician at the Institute for Medical Biometry, University Hospital Heidelberg, Germany. Randomization considered the number of practices in each PCN, and based on the expected number of practices, aimed for comparable numbers of practices in each intervention arm. Due to intervention components to be offered on PCN level, randomization on practice level was not feasible. For administrative reasons, the focus was on cases in patients insured by AOK health insurance and registered within a specific healthcare delivery program (defined by German law $\S 140 a$ SGB V a.F. and $\S 140 a$ Abs. 1. S. 2 Alt. 1 SGB V n.F). At baseline, approximately 40,000 patients with AOK health insurance were registered in 196 participating primary care practices in the 14 PCNs. Medical specialties covered by participating physicians included general practice (largest group), otolaryngology, pediatrics, urology and gynecology. Observed cases comprised patients who consulted a physician in participating PCNs or in the matched clusters for one of the following reasons: acute upper respiratory tract infection (URTI), acute bronchitis, acute sinusitis, tonsillitis, otitis media, urinary tract infection (UTI), community aquired pneumonia. All diagnoses were based on physician-recorded ICD-10 codes and prescribing information in the administrative data provided by the health insurer for quarterly reimbursement periods which were linked by the pseudonymized individual insurance number. Cross-validation of recorded codes with patient charts was not possible due to data confidentiality regulations. Each physician-recorded ICD-10 code for defined index diagnoses in the observed quarters represents a case, and each patient could produce multiple cases. Diagnoses for other diseases and diagnoses that warrant an antibiotic therapy were not considered. (Appendix A, Supplementary File 3, Table A5 details diagnoses and related ICD-10 codes and Table A6 provides a list of disregarded diagnoses.) Note that prescribing information was derived from quarterly claims data provided by the health insurer. Patients were not actively recruited, but gave consent to their data being included for analysis.

\section{Measures}

The primary outcome references established indicators of the European Surveillance of Antimicrobial Consumption Network (ESAC-Net) [42] which were tailored to the specifics of ARena. It was defined as the percentage of patient cases with acute non-complicated infections receiving an antibiotic prescription without pathogen detection when consulting primary care practices (cases with acute bronchitis (age 18-75), sinusitis ( $>18$ years), otitis media ( $>2$ years), URTI $(>1$ year), or tonsillitis $(>1$ year)). Evaluation was based on quarterly claims data. The intervention period was planned for 24 months and 
reduced to 21 months (Q4 2017 to Q2 2019) due to delays in component development. As indicated by the study protocol [24], points of measurement were at baseline (Q3 2016 Q2 2017) and at the end of intervention period (Q3 2018 - Q2 2019). Diagnoses for streptococcal tonsilitis and other pathogen-caused acute forms of tonsillitis that warrant antibiotic therapy were not considered.

SOs examined in this study refer to the percentage of cases with (SO 1) acute noncomplicated infections receiving a quinolone prescription when consulting primary care practices (cases with acute bronchitis (age 18-75), sinusitis (>18), otitis media (>2 ), URTI ( $>1$ year), or tonsillitis (age $>1)$ ); (SO 2) acute URTI ( $>1$ year) receiving a prescription for recommended antibiotics (amoxicillin); (SO 3) acute bronchitis (age 18-75) receiving a prescription for recommended antibiotics (amoxicillin, tetracycline, macrolides); (SO 4) sinusitis ( $>18$ years) receiving a prescription for recommended antibiotics (amoxicillin, cefuroxime); (SO 5) tonsillitis (>1 year) receiving a prescription for recommended antibiotics (penicillin, erythromycin); and (SO 6) otitis media ( $>2$ years) receiving a prescription for recommended antibiotics (amoxicillin, erythromycin, cefuroxime).

The ARena study protocol also defined SOs referring to cystitis and communityaquired pneumonia which were not considered here due to a very small numbers of cases. Specifications for patient age in all observed sub-groups adhere to study protocol [24]. All outcomes reported here relate to ICD-10 coded consultations in primary care and prescribing of systemic antibiotics and indication-specific prescribing of currently guidelinerecommended antimicrobials $[43,44]$. Recommended antibiotics were categorized based on existing evidence-based clinical guidelines developed by the German College of General Practitioners and Family Physicians (DEGAM) [43] and the Association of the Scientific Medical Societies in Germany (AWMF) [44]. (See Appendix A, Supplementary File 3, Table A7 for currently recommended and alternative antibiotics.)

The following patient-related sociodemographic, disease, and treatment characteristics were provided in the claims data and as indicated by the study protocol [24] included for analysis: age, sex, Charlson comorbidity index (CCI) (predicts 1-year survival in patients based on sum of relevant comorbidities) [45, 46], nationality (missing values were aggregated to 'other'). For the primary care practices, type of location (urban, urbanized, countryside), type of practice (single or group) and medical specialty group are documented.

\section{Data analysis}

Data were analyzed using the statistical software $\mathrm{R}$ version 3.6 .3 to compare four baseline quarters (Q3 2016 - Q2 2017) to four quarters at the end of the intervention (Q3 2018 - Q2 2019) for the intervention arms, between arms, and in comparison to the matched clusters in standard care regarding the defined outcomes. The primary and secondary outcomes, documented data referring to patient and disease characteristics, treatment data, and distribution of medical specialty group were first analyzed descriptively stratified by intervention arm. For categorical variables, absolute and relative frequencies are provided. Note that patient and disease characteristics, treatment data and practice characteristics differ between outcomes as considered cases are defined for each outcome by the respective disease and prescribing of antibiotics. Confirmatory analysis of the primary outcome was conducted based on the Intention-To-Treat (ITT) population. A threestep, structured hierarchical test procedure was applied [47] to ensure a global significance level of $5 \%$ and assess the following hypotheses regarding the pre-post intervention comparison of the percentage of cases with acute non-complicated infections receiving antibiotic prescription when consulting primary care practices:

Step 1: Percentage of cases in intervention arms II and III are each lower post intervention.

Step 2: Percentage of cases in intervention arm I is lower post intervention.

Step 3: Compared to intervention arm I, the percentage is lower in intervention arms II and III each. 
In Step 1, the pre-post-comparisons in intervention arms II and III were tested using a $2.5 \%$ significance level (Bonferroni correction). If at least one pre-post-comparison was significant, Step 2 was conducted (significant difference in one arm in step 1: $2.5 \%$ significance level, significant difference in both arms: 5\% significance level). In case the prepost-comparison in Step 2 was significant, the pre-post-comparisons between intervention arms were tested in Step 3 to examine intervention effectiveness. Logistic mixed effects models were used to explore the primary objective in the hierarchical procedure. The models considered the nested structure of the data with multiple cases per patient and patients nested in practices by including a random effect in the logistic mixed effects model for patients and practices. As fixed effects, timepoint (pre/post), gender, and age group $(<18,18-65,>65$ years) were included in step 1 and 2 . In step 3 , the intervention arm was added as fixed effect.

The primary outcome was additionally analysed comparing the three intervention arms to matched standard care using a logistic mixed effects model and taking the clustered structure into account. Standard care was matched on practices level by using a propensity score matching based on a logistic regression model under consideration of the matching variables specialist group, baseline number of cases, postal code (first 3 digits), and region. Considered fixed effects in that model are group (intervention arms vs. matched standard care), time-point (pre/post), gender, age, CCI as indication of health status, nationality, region, town/countryside. Secondary outcomes were analyzed using mixed logistic regression models comparing intervention arms with the matched standard care. All p-values for secondary analysis are of explorative nature. Findings reported here focus on prescribing of antibiotics for upper respiratory tract infections.

\title{
5. Conclusions
}

Significantly reduced antibiotic prescribing for non-complicated infections in the intervention arms and compared to matched standard care show the impact of the implementation program. Further research should explore which interventions can increase the effects.

\author{
Abbreviations \\ ARTI - acute respiratory tract infections \\ AWMF - Association of the Scientific Medical Societies in Germany \\ CAP - community acquired pneumonia \\ $\mathrm{CI}$ - confidence interval \\ CCI - Charlson comorbidity Index \\ CDSS - Computerized Decision Support System \\ DDD - defined daily dose \\ DEGAM - German College of General Practitioners and Family Physicians \\ ESAC-Net - European Surveillance of Antimicrobial Consumption Network \\ GP - general practitioners \\ ICD - International Classification of Diseases \\ $\mathrm{OR}$ - odds ratio \\ PCNs - primary care networks \\ $\mathrm{Q}$ - quarter \\ SO - Secondary outcome \\ URTI - upper respiratory tract infections
}

Supplementary Materials: The following are available online at www.mdpi.com $/ x x x / s 1$, 


\author{
Supplementary File 1, Table A1-A2 \\ Supplementary File 2, Table A3-A4 \\ Supplementary File 3, Table A5-A7
}

\begin{abstract}
Author Contributions: RPD, DK, MK and MW drafted and prepared the manuscript. DK, RPD, and MW contributed to concept and design of this study. DK, RPD, MW, PKK and EA collaborated on the construction of the statistical analysis plan. DK analyzed the claims data. DK, RPD, MK and MW interpreted the data. PKK and EA were responsible for data delivery. JB and VW contributed to development and design of the trial and supported recruitment of PCNs. PKK, EA, JB, VW and JS provided their expertise. JS contributed to the design of the trial and was overall principal investigator of the ARena project. All authors provided substantial comments and approved the final version of the manuscript.
\end{abstract}

RPD - Regina Poss-Doering; DK - Dorothea Kronsteiner; MK - Martina Kamradt; PKK - Petra Kaufmann-Kolle; EA - Edith Andres; JB - Julian Bleek; VW - Veit Wambach; MW - Michel Wensing; JS Joachim Szecsenyi

Conceptualization, Regina Poss-Doering, Dorothea Kronsteiner, Petra Kaufmann-Kolle, Edith Andres, Veit Wambach, Julian Bleek, Michel Wensing and Joachim Szecsenyi; Data curation, Petra Kaufmann-Kolle, Edith Andres and ARena Study group; Formal analysis, Regina Poss-Doering and Dorothea Kronsteiner; Funding acquisition, ARena Study group and Joachim Szecsenyi; Investigation, Regina Poss-Doering and Michel Wensing; Methodology, Regina Poss-Doering, Dorothea Kronsteiner and Michel Wensing; Project administration, Regina Poss-Doering, Martina Kamradt, Petra Kaufmann-Kolle, Edith Andres and ARena Study group; Resources, ARena Study group; Supervision, Michel Wensing and Joachim Szecsenyi; Validation, Dorothea Kronsteiner and Martina Kamradt; Visualization, Regina Poss-Doering, Dorothea Kronsteiner and Martina Kamradt; Writing - original draft, Regina Poss-Doering and Dorothea Kronsteiner; Writing - review \& editing, Regina Poss-Doering, Dorothea Kronsteiner, Martina Kamradt, Petra Kaufmann-Kolle, Edith Andres, Veit Wambach, Julian Bleek, Michel Wensing and Joachim Szecsenyi.

Funding: The ARena study received funding from the Innovation Committee at the Federal Joint Committee (G-BA), Berlin (01NVF16008). The funder had no role in the design, data-collection, dataanalysis, interpretation, or writing of the paper.

Institutional Review Board Statement: The study was conducted according to the guidelines of the Declaration of Helsinki, and approved by the Ethics Committee of the Medical Faculty of Heidelberg University (S-353/2017, 03Aug2017). Trial registration: ISRCTN, ISRCTN58150046

Informed Consent Statement: Informed consent was obtained from all participants, confidentiality and anonymity were ensured throughout the study. Participants all gave written informed consent for participation and publication of findings.

Data Availability Statement: All analyses generated for this study are included in this published article and its supplementary information files 1-3 (Appendix A). The original datasets that support the findings of this study are not publicly available due to restrictions stipulated by German law and the data provider. AOK can be contacted for access to the data.

Acknowledgments: The authors would like to thank the aQua Institute and their staff for taking the lead in the ARena consortium. In particular, we thank Linda Barnewold, aQua Institut, for supporting data management in this study and Andreas Gutscher, Gitta Friedrichs und Yvonne Giesler, aQua Institut, for their administrative support. We would also like to thank all involved ARena project partners for their advice, contributions and overall support in conducting the ARena study: Agency of German Practice Based Physicians (Agentur deutscher Arztnetze), statutory health insurers AOK Bayern, AOK Rheinland-Hamburg, and Federal AOK Association (AOK Bundesverband), and the Bavarian Association of Statutory Health Insurance Physicians (KV Bayerns).

**ARena-study group: Joachim Szecsenyi, Michel Wensing, Martina Kamradt, Regina Poss-Doering, Dept. of General Practice and Health Services Research, University Hospital Heidelberg; Dorothea Kronsteiner, IMBI Institute for Medical Biometry, Heidelberg; Petra Kaufmann-Kolle, Edith Andres, aQua Institut, Goettingen; Non-author contributors: Joerg Lindenthal, QuE Nuremberg; Alexander Günter, statutory health insurer AOK Bavaria (AOK Bayern), Mara Plambeck and Witali Schleiz statutory health insurer AOK Rheinland/Hamburg, Lutz Bader, Hanna Schürkämper and Sandra 
Hérault, Bavarian Association of Statutory Health Insurance Physicians (KV Bayerns) Bavarian Association of Statutory Health Insurance Physicians (KV Bayerns) served as scientific advisors throughout the ARena study.

Conflicts of Interest: JS is founder, shareholder and managing director of the aQua Institut, Goettingen and Medical Director at the Department of General Practice and Health Services Research, University Hospital Heidelberg, Germany. Edith Andres is co-founder and shareholder of the aQua Institut, Goettingen. All other authors declare that they have no conflicts of interests.

\section{References}

1. 1. German Federal Ministry of Health. Bundesministerium für Gesundheit. DART 2020 - Antibiotika-Resistenzen bekämpfen zum Wohl von Mensch und Tier. Berlin: Bundesministerium für Gesundheit; 2015.

2. 2. Federal Office of Consumer Protection and Food Safety P-E-GfCeV. Germap 2015: Antimicrobial Resistance and Consumption; Report on the consumption of antimicrobials and the spread of antimicrobial resistance in human and veterinary medicine in Germany. Rheinbach: Antiinfectives Intelligence; 2016.

3. 3. European Centre for Disease Prevention and Control. Surveillance of antimicrobial resistance in Europe 2018. Stockholm2019 [Available from: https://www.ecdc.europa.eu/sites/default/files/documents/surveillance-antimicrobial-resistanceEurope-2018.pdf.

4. 4. European Centre for Disease Prevention Control. Antimicrobial resistance surveillance in Europe. Annual report of the European Antimicrobial Resistance Surveillance Network (EARS-Net) 2015. Stockholm: ECDC; 2017 [27.07.2021]. Available from: https://www.ecdc.europa.eu/sites/default/files/media/en/publications/Publications/antimicrobial-resistance-europe2015.pdf.

5. 5. Kraus EM, Pelzl S, Szecsenyi J, Laux G. Antibiotic prescribing for acute lower respiratory tract infections (LRTI) - guideline adherence in the German primary care setting: An analysis of routine data. PloS one. 2017;12(3):e0174584.

6. 6. German Federal Ministry of Health. Bundesministerium für Gesundheit. DART 2020 - Antibiotika-Resistenzen bekämpfen zum Wohl von Mensch und Tier. Berlin: German Federal Ministry of Health; 2015.

7. 7. Anthierens S, Tonkin-Crine S, Cals JW, Coenen S, Yardley L, Brookes-Howell L, et al. Clinicians' Views and Experiences of Interventions to Enhance the Quality of Antibiotic Prescribing for Acute Respiratory Tract Infections. Journal of General Internal Medicine. 2015;30(4):408-16.

8. 8. Meeker D, Linder JA, Fox CR, Friedberg MW, Persell SD, Goldstein NJ, et al. Effect of Behavioral Interventions on Inappropriate Antibiotic Prescribing Among Primary Care Practices: A Randomized Clinical Trial. JAMA. 2016;315(6):562-70.

9. 9. Ivers N, Jamtvedt G, Flottorp S, Young JM, Odgaard-Jensen J, French SD, et al. Audit and feedback: Effects on professional practice and healthcare outcomes. The Cochrane database of systematic reviews. 2012(6):CD000259.

10. 10. Wensing M, Broge B, Riens B, Kaufmann-Kolle P, Akkermans R, Grol R, et al. Quality circles to improve prescribing of primary care physicians. Three comparative studies. Pharmacoepidemiol Drug Saf. 2009;18(9):763-9.

11. 11. Kochling A, Loffler C, Reinsch S, Hornung A, Bohmer F, Altiner A, et al. Reduction of antibiotic prescriptions for acute respiratory tract infections in primary care: a systematic review. Implement Sci. 2018;13(1):47.

12. 12. Agentur deutscher Arztnetze (AdA). Ueber Netze. Was sind Arztnetze? : Agentur deutscher Arztnetze; 2014 [ 16.06.2021]. Available from: http://deutsche-aerztenetze.de/ueber_netze/was sind arztnetze.php.

13. 13. Cunningham FC, Ranmuthugala G, Plumb J, Georgiou A, Westbrook JI, Braithwaite J. Health professional networks as a vector for improving healthcare quality and safety: a systematic review. BMJ Qual Saf. 2012;21(3):239-49.

14. 14. Freund T, Everett C, Griffiths P, Hudon C, Naccarella L, Laurant M. Skill mix, roles and remuneration in the primary care workforce: Who are the healthcare professionals in the primary care teams across the world? International Journal of Nursing Studies. 2015;52(3):727-43.

15. 15. Chapman SA, Blash LK. New Roles for Medical Assistants in Innovative Primary Care Practices. Health Serv Res. 2017;52 Suppl 1(Suppl 1):383-406.

16. 16. Tonkin-Crine SK, Tan PS, van Hecke O, Wang K, Roberts NW, McCullough A, et al. Clinician-targeted interventions to influence antibiotic prescribing behaviour for acute respiratory infections in primary care: An overview of systematic reviews. The Cochrane database of systematic reviews. 2017;9:CD012252.

17. 17. Little P, Stuart B, Francis N, Douglas E, Tonkin-Crine S, Anthierens S, et al. Effects of internet-based training on antibiotic prescribing rates for acute respiratory-tract infections: A multinational, cluster, randomised, factorial, controlled trial. The Lancet. 2013;382(9899):1175-82.

18. 18. Rohrbasser A, Harris J, Mickan S, Tal K, Wong G. Quality circles for quality improvement in primary health care: Their origins, spread, effectiveness and lacunae- A scoping review. PLoS One. 2018;13(12):e0202616.

19. 19. Chauhan BF, Jeyaraman M, Mann AS, Lys J, Skidmore B, Sibley KM, et al. Behavior change interventions and policies influencing primary healthcare professionals' practice-an overview of reviews. Implementation Science. 2017;12(1):3.

20. 20. Holstiege J, Mathes T, Pieper D. Effects of computer-aided clinical decision support systems in improving antibiotic prescribing by primary care providers: a systematic review. J Am Med Inform Assoc. 2015;22(1):236-42. 
21. 21. Gonzales R, Anderer T, McCulloch CE, Maselli JH, Bloom FJ, Graf TR, et al. A cluster randomized trial of decision support strategies for reducing antibiotic use in acute bronchitis. JAMA internal medicine. 2013;173(4):267-73.

22. 22. Terry A. Do clinical decision support systems reduce inappropriate antibiotic prescribing for acute bronchitis? $2017 ; 21$.

23. 23. Mann D, Hess R, McGinn T, Richardson S, Jones S, Palmisano J, et al. Impact of Clinical Decision Support on Antibiotic Prescribing for Acute Respiratory Infections: a Cluster Randomized Implementation Trial. J Gen Intern Med. 2020;35(Suppl 2):788-95.

24. 24. Kamradt M, Kaufmann-Kolle P, Andres E, Brand T, Klingenberg A, Glassen K, et al. Sustainable reduction of antibioticinduced antimicrobial resistance (ARena) in German ambulatory care: study protocol of a cluster randomised trial. Implementation Science. 2018;13(1):23.

25. 25. Davey P, Marwick CA, Scott CL, Charani E, McNeil K, Brown E, et al. Interventions to improve antibiotic prescribing practices for hospital inpatients. Cochrane Database of Systematic Reviews. 2017(2).

26. 26. Poss-Doering R, Kühn L, Kamradt M, Stürmlinger A, Glassen K, Andres E, et al. Fostering Appropriate Antibiotic Use in a Complex Intervention: Mixed-Methods Process Evaluation Alongside the Cluster-Randomized Trial ARena. Antibiotics (Basel, Switzerland). 2020;9(12).

27. 27. Poss-Doering R, Kamradt M, Stuermlinger A, Glassen K, Kaufmann-Kolle P, Andres E, et al. The complex phenomenon of dysrational antibiotics prescribing decisions in German primary healthcare: a qualitative interview study using dual process theory. Antimicrobial Resistance \& Infection Control. 2020;9(1):6.

28. 28. Andres E, Szecsenyi J, Garbe K, Hartmann J, Petruschke I, Schulz M, et al. Rational Use of Antibiotics: Impulses for Primary Care (a Symposium Report). Zeitschrift für Allgemeinmedizin ZFA 2020;96 (3):109-15.

29. 29. Cunningham FC, Ranmuthugala G, Plumb J, Georgiou A, Westbrook JI, Braithwaite J. Health professional networks as a vector for improving healthcare quality and safety: A systematic review. BMJ quality \& safety. 2012;21(3):239-49.

30. 30. Valente TW, Palinkas LA, Czaja S, Chu K-H, Brown CH. Social network analysis for program implementation. PloS one. 2015;10(6):e0131712.

31. 31. Poss-Doering R, Kamradt M, Glassen K, Andres E, Kaufmann-Kolle P, Wensing M. Promoting rational antibiotic prescribing for non-complicated infections: understanding social influence in primary care networks in Germany. BMC Family Practice. 2020;21(1):51.

32. 32. Poss-Doering R, Kronsteiner D, Kamradt M, Andres E, Kaufmann-Kolle P, Wensing M, et al. Antibiotic prescribing for acute, non-complicated infections in primary care in Germany: baseline assessment in the cluster randomized trial ARena. BMC Infectious Diseases. 2021;21(1):877.

33. 33. Kamtsiuris P, Atzpodien K, Ellert U, Schlack R, Schlaud M. Prävalenz von somatischen Erkrankungen bei Kindern und Jugendlichen in Deutschland. Bundesgesundheitsblatt - Gesundheitsforschung - Gesundheitsschutz. 2007;50(5):686-700.

34. 34. Grobe TG, Dörning H, FW S. BARMER GEK Arztreport. St. Augustin: Asgard; 2011.

35. 35. Coker TR, Chan LS, Newberry SJ, Limbos MA, Suttorp MJ, Shekelle PG, et al. Diagnosis, microbial epidemiology, and antibiotic treatment of acute otitis media in children: a systematic review. Jama. 2010;304(19):2161-9.

36. 36. Britten N. Patients' expectations of consultations. Bmj. 2004;328(7437):416-7.

37. 37. Mangione-Smith R, McGlynn EA, Elliott MN, Krogstad P, Brook RH. The relationship between perceived parental expectations and pediatrician antimicrobial prescribing behavior. Pediatrics. 1999;103(4 Pt 1):711-8.

38. 38. Venekamp RP, Sanders SL, Glasziou PP, Del Mar CB, Rovers MM. Antibiotics for acute otitis media in children. Cochrane Database Syst Rev. 2015;2015(6):Cd000219.

39. 39. Holstiege J, Schulz M, Akmatov M, Kern W, Steffen A, Bätzing J. The decline in outpatient antibiotic use - an analysis of nationwide prescription data from 2010 to 2018. Deutsches Ärzteblatt. 2020;117:679-86.

40. 40. De Sutter AI, De Meyere MJ, De Maeseneer JM, Peersman WP. Antibiotic prescribing in acute infections of the nose or sinuses: a matter of personal habit? Fam Pract. 2001;18(2):209-13.

41. 41. Hueber S, Kuehlein T, Gerlach R, Tauscher M, Schedlbauer A. "What they see is what you get": Prescribing antibiotics for respiratory tract infections in primary care: Do high prescribers diagnose differently? An analysis of German routine data. PLoS One. 2017;12(12):e0188521.

42. 42. Adriaenssens N, Coenen S, Versporten A, Muller A, Minalu G, Faes C, et al. European Surveillance of Antimicrobial Consumption (ESAC): outpatient antibiotic use in Europe (1997-2009). J Antimicrob Chemother. 2011;66 Suppl 6:vi3-12.

43. 43. (DEGAM). GCoGPaFP. Leitlinien der DEGAM. [Available from: https://www.degam.de/degam-leitlinien-379.html.

44. 44. Association of the Scientific Medical Societies in Germany (AWMF). Aktuelle Leitlinien (Current guideliens)25.01.2021 27.01.2021]. Available from: https://www.awmf.org/leitlinien/aktuelle-leitlinien.html.

45. 45. Charlson ME, Pompei P, Ales KL, MacKenzie CR. A new method of classifying prognostic comorbidity in longitudinal studies: development and validation. J Chronic Dis. 1987;40(5):373-83.

46. 46. Quan H, Sundararajan V, Halfon P, Fong A, Burnand B, Luthi JC, et al. Coding algorithms for defining comorbidities in ICD-9-CM and ICD-10 administrative data. Med Care. 2005;43(11):1130-9.

47. 47. Westfall PH, Krishen A. Optimally weighted, fixed sequence and gatekeeper multiple testing procedures. Journal of Statistical Planning and Inference. 2001;99(1):25-40.

48. 
